\title{
Gefäßvarianten erkennen und nachschlagen: Schlüsselreferenz und neue App
}

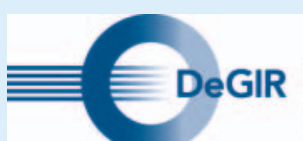

DEUTSCHE GESELLSCHAFT FÖR INTERVENTIONELLE RADIOLOGIE

UND MINIMAL-INVASIVE THERAPIE

in der Deutschen Röntgengesellschaft e.V.

Eine gute Kenntnis über Gefäßvarianten ist nicht nur für den Interventionalisten oder die Interventionalistin wichtig, auch für den rein diagnostisch tätigen Radiologen oder Radiologin hat diese eine hohe Relevanz. Ob es sich hierbei nun um die retroaortal verlaufende Nierenvene vor Aortenchirurgie, die linksseitige Vena cava superior mit dem nicht-typisch liegenden zentralen Venenkatheter (ZVK) oder um die aberrante rechte Leberarterie vor geplanter Leberintervention handelt, so ist das Erkennen und die Beschreibung als Normvariante im Befund essentiell. Das Übersehen von Gefäßvarianten kann fatale Konsequenzen haben. So denke man beispielsweise daran, wie die „Corona mortis“ zu ihrem Namen kam oder die möglichen Komplikationen von HNO-chirurgischen Eingriffen bei aberrantem Verlauf der „Arteria carotis interna“ oder bei „kissing carotids“. Als Interventionalist/-in hat man es schwer, wenn man z. B. eine Notfall-Embolisation der „Arteria gastroduodenalis“ bei aktiver Ulcusblutung durchführen soll und sich die Arterie nicht an ihrem gewohnten Platz befindet. Wo muss man sie suchen? Bis vor wenigen Jahren musste man noch mühsam in einzelnen Nachschlagewerken und Veröffentlichungen nach der Beschreibung von Gefäßvarianten suchen, da es keine universelle Zusammenfassung in Form eines Atlas‘ gab.

Prof. Dr. Frank Wacker, Direktor des Instituts für Diagnostische und Interventionelle Radiologie der Medizinischen Hochschule Hannover, hat Abhilfe geschaffen, indem er das von den Anato- men Prof. Dr. Herbert Lippert und Prof. Dr. Reinhard Pabst im Jahre 1985 herausgegebene Buch mit radiologischen Bildbeispielen als universelles Nachschlagewerk für arterielle Gefäßvarianten unter der Mitarbeit seines radiologischen Teams neu herausgebracht hat. Die Gefäßvarianten sind durch schematische Zeichnungen und zusätzliche Bildbeispiele aus der radiologischen Bildgebung veranschaulicht.

Zudem gibt es seit diesem Jahr eine neue App mit dem Titel „Vascular Variants“, die als Nachschlagewerk nicht nur für arterielle Gefäßvarianten dient. Dr. Mareike Franke, Interventionelle Radiologin in der Klinik Dr. Hancken, Stade, hat aus verschiedenen verfügbaren Quellen in über 800 anatomischen Zeichnungen Varianten von Arterien, Venen, Pankreasgängen und Gallengängen zusammengetragen, die dann mittels weniger Klicks nachgeschlagen werden können. 


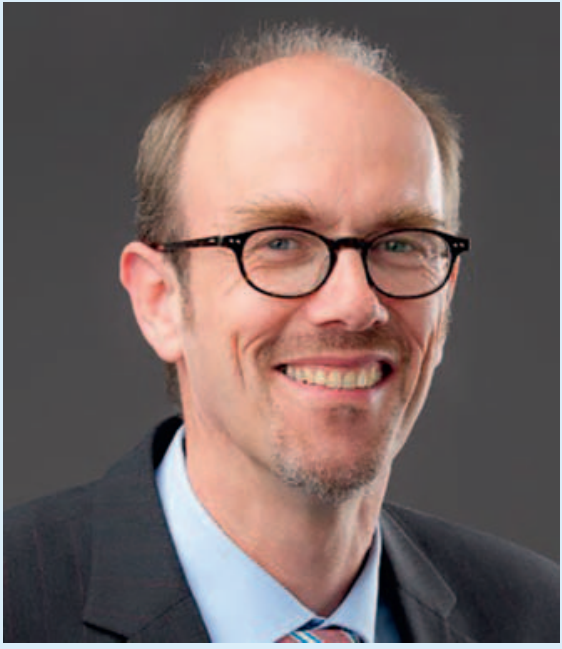

Prof. Dr. Frank Wacker

Herr Prof. Wacker, wie sind Sie zum Experten für dieses spezielle Thema geworden?

Prof. Dr. Frank Wacker: Seit ich in der Angiografie arbeiten durfte, hat mich das Thema interessiert. Ich denke, dass alle in der Interventionellen Radiologie tätigen Expertinnen und Experten für Gefäßvarianten sind. Das zeigt ja nicht zuletzt auch die tolle App von Frau Dr. Franke, die die Notwendigkeit gesehen hat, die Information nahe am Patienten und der Patientin parat zu haben. Durch die immer besseren Möglichkeiten der CTA und MRA ist das Thema jedoch auch, über die Interventionelle Radiologie und die Chirurgie hinaus, für die Diagnostik relevant.

Was gab den Ausschlag bzw. wann hatten Sie die Idee eine Schlüsselreferenz für arterielle Gefäßvarianten zu schreiben?

Wacker: Während meiner Weiterbildung in der Radiologie des Klinikum Steglitz der FU Berlin habe ich das Buch von Lippert und Pabst sehr häufig genutzt. Es hat mir oft geholfen, zu verstehen, welche anatomische Variation ich gerade angiografiert habe. Nach meiner Berufung an die Medizinische Hochschule Hannover habe ich die beiden Anatomen, beide $\mathrm{MHH}$ Alumni, dann persönlich kennen gelernt. Mit Hilfe vieler Mitarbeitender der Radiologie und Neuroradiologie der $\mathrm{MHH}$ entstand dann die Neuauflage ihres Buches, angereichert mit radiologischen Abbildungen. Ich nutze das Buch weiterhin sehr häufig, wenn es um die i.a.-

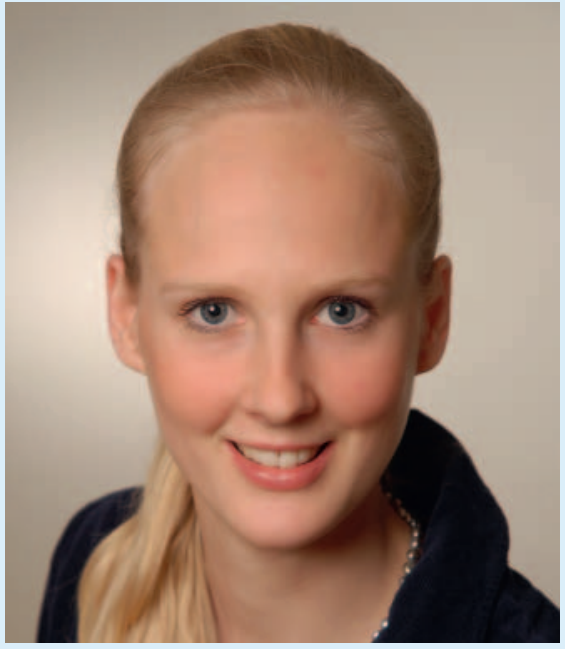

Dr. Mareike Franke

Therapie von Lebertumoren geht und wir zum Beispiel die Aa. gastricae suchen. Natürlich haben wir mit dem C-Arm-CT inzwischen aber auch hervorragende Möglichkeiten, in der Angiografie Gefäße und Parenchym dreidimensional in hoher Auflösung darzustellen und so Komplikationen zu vermeiden.

Frau Dr. Franke, wie sind Sie auf die Idee gekommen, Gefäßvarianten als Nachschlage-App zusammenzutragen? Was war Ihre Motivation?

Dr. Mareike Franke: Auch ich habe mich schon sehr früh für Gefäßvarianten interessiert. Bereits im Studium habe ich gerne das Kleingedruckte in den Anatomiebüchern über die Normabweichungen gelesen. Natürlich kenne ich die neu herausgebrachte hervorragende Schlüsselreferenz von den Herren Professoren Wacker, Lippert und Pabst. Das Exemplar in unserer Abteilung ist jedoch immer verschwunden, wenn man mal etwas nachschlagen will. Irgendwer hat es sich immer „ausgeliehen“, da man das Buch insbesondere beim Befunden super als Nachschlagewerk nutzen kann. Somit habe ich mir gedacht, dass es doch praktisch wäre, ein Nachschlagewerk auf dem Handy als App ganz unkompliziert immer zur Verfügung zu haben. Und da ich 2019 schon eine App über Abkürzungen und Akronyme in der Interventionellen Radiologie herausgebracht habe, dachte ich mir, das mache ich jetzt!
Es gibt in der Literatur je nach Quelle ganz unterschiedliche Angaben bezüglich der Inzidenz von Gefäßvarianten. Herr Prof. Wacker, wie sind Sie beim Verfassen des Buches also vorgegangen?

Wacker: Die Angaben der Häufigkeiten der arteriellen Varianten sind in der Tat eine große Herausforderung. In der Literatur existieren Ergebnisse anatomischer Dissektionen, aber auch Erkenntnisse aus angiografischen Untersuchungen ex vivo und in vivo mit unterschiedlichen Techniken, bei Kranken und Gesunden, aus unterschiedlichen Regionen der Welt. Dies verursacht eine Verzerrung der Stichproben. Wir haben versucht, vorwiegend die Zahlen aus größeren Studien zusammenzufassen, aber für einige Gefäßregionen liegen nur Fallberichte über seltene Varianten vor.

Frau Dr. Franke, wie sind Sie bei der Erstellung der App vorgegangen?

Franke: Als erstes musste ich mir natürlich überlegen, wie ich die Gefäßvarianten darstellen wollte. Da ich nicht nur Gefäßvarianten in die App aufnehmen wollte, sondern auch Abbildungen von Arealen, die in den gängigen Anatomieatlanten nicht so gut beleuchtet werden - als Beispiel zu nennen sind hier die kleinen Kollateralen zwischen dem Stromgebiet der Arteria carotis interna und externa auf Höhe der Schädelbasis -, habe ich mir überlegt, dass ich die Abbildungen selber zeichnen möchte, um auch solche Regionen in möglichst einfacher Weise begreifbar zu machen. Wie auch in der Schlüsselreferenz habe ich Häufigkeitsangaben zu den Varianten gemacht - soweit ich sie denn in der Literatur gefunden habe - und habe mich hier an den Studien mit größeren Fallzahlen oder an den Klassifikationen orientiert, die sich in der radiologischen Community durchgesetzt haben. Letztendlich bin ich die Literatur durchgegangen und habe mir - wenn möglich aus Primärquellen - die Informationen zusammengesucht und dann die entsprechenden Zeichnungen angefertigt. Diese habe ich dann zusammen mit den entsprechenden Erklärungen in eine Datenbank eingefügt, die der App zugrunde liegt. Neben arteriellen Varianten sind übrigens auch Varianten von Venen, Gallengängen oder Pankreasgängen in der App nachzuschlagen. 
Frau Dr. Franke, wird die App laufend aktualisiert oder erweitert und falls ja: Was ist für die nächsten Updates geplant?

Franke: Die App soll in regelmäßigen Abständen aktualisiert werden, denn man findet ja immer wieder neue Gefäßvarianten, die man noch mit in die Datenbank aufnehmen kann. Zudem funktioniert der Suchalgorithmus in meiner App aktuell so, dass man den Namen des Gefäßes auf Latein oder Englisch eingeben kann und dann zu den entsprechenden Abbildungen über Varianten oder die Normalanatomie kommt. Das heißt, man muss schon wissen, was man sucht. In einem zukünftigen Update möchte ich gerne diesen Suchalgorithmus erweitern, sodass man in der App die verschiedenen anatomischen Regionen und Gefäßtypen nach Variationen durchstöbern kann. Selbstverständlich bin ich auch immer offen für Verbesserungsvorschläge von Kolleginnen und Kollegen und würde mich freuen, wenn ich Rückmeldung zur App bekommen würde - eine E-Mail-Adresse ist im Impressum der App angegeben. Darüber hinaus bin ich auch offen für weitere Projekte zu Gefäßvarianten.
Herr Prof. Wacker, wird es eine zweite Auflage Ihres Buches geben oder was sind Ihre nächsten Projekte auf diesem Gebiet?

Wacker: Aktuell ist das noch nicht geplant. Großartig wäre es natürlich, wenn Kolleginnen und Kollegen in der DeGIR, angeregt durch die Nutzung der App oder durch die Lektüre des Atlas', uns CTA-, MRA- oder DSA-Bilder von anatomischen Varianten zusenden. Dies würde uns sicher motivieren, an eine erneute Auflage des Buches zu denken, denn nicht von allen Varianten haben wir radiologische Bilder gefunden. Gerne binden wir auch Expertinnen und Experten für bestimmte Gefäßterritorien mit entsprechendem Bildmaterial ein. Wir freuen uns zudem immer über die Zusendung von neuen Publikationen zu arteriellen Varianten. Ihre Frage zur Häufigkeit nochmal aufgreifend sind wir natürlich auch sehr gerne bereit, Diskussionen über Häufigkeiten und klinische Relevanz von Varianten zu führen.

\section{Vielen Dank für das Gespräch!}

Das Buch ist deutscher und englischer Sprache im Thieme-Verlag erhältlich: Arterial Variations in Humans: Key Reference for Radiologists and Surgeons 2017

ISBN: 978-3-13-200471-9

Atlas der arteriellen Variationen: Klassifikation und Häufigkeit 2018

ISBN: 978-3-13-172961-3

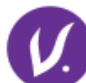

Die App steht kostenfrei zum Download zur Verfügung:

Vascular Variants: $800+$ anatomical illustrations for vascular variants at your fingertips.

Android: https://play.google.com/ store/apps/details?id=com.n53stu dios.vascularvariations iOS https://apps.apple.com/app/id 1533515801

Das Interview führte Dr. Kerstin Westphalen, DeGIR-Lenkungsgruppe Öffentlichkeitsarbeit. 